\title{
JUDE: An Ultraviolet Imaging Telescope Pipeline
}

\author{
Jayant Murthy $^{\mathrm{a}}$, P. T. Rahna ${ }^{\mathrm{b}}$, Firoza Sutaria ${ }^{\mathrm{a}}$, Margarita Safonova ${ }^{\text {c,* }}$, S. B. Gudennavar ${ }^{\mathrm{b}}$, \\ S. G. Bubbly \\ ${ }^{a}$ Indian Institute of Astrophysics, Bengaluru \\ ${ }^{b}$ Department of Physics, Christ University, Bengaluru \\ ${ }^{c}$ M. P. Birla Institute of Fundamental Research, Bengaluru
}

\begin{abstract}
The Ultraviolet Imaging Telescope (UVIT) was launched as part of the multi-wavelength Indian AstroSat mission on 28 September, 2015 into a low Earth orbit. A 6-month performance verification (PV) phase ended in March 2016, and the instrument is now in the general observing phase. UVIT operates in three channels: visible, near-ultraviolet (NUV) and far-ultraviolet (FUV), each with a choice of broad and narrow band filters, and has NUV and FUV gratings for low-resolution spectroscopy. We have written a software package (JUDE ) to convert the Level 1 data from UVIT into scientifically useful photon lists and images. The routines are written in the GNU Data Language (GDL) and are compatible with the IDL software package. We use these programs in our own scientific work, and will continue to update the programs as we gain better understanding of the UVIT instrument and its performance. We have released JUDE under an Apache License.
\end{abstract}

Keywords: ultraviolet, UVIT, astronomical software, data analysis

\section{Introduction}

The Ultraviolet Imaging Telescope (UVIT) was first proposed as part of the multi-wavelength $A s$ troSat mission in the late $20^{\text {th }}$ century (Pati and Rao, 1998, Pati, 1999) and was launched on 28 September, 2015 into a low Earth orbit $(650 \mathrm{~km}$, $6^{\circ}$ inclination) by an ISRO (Indian Space Research Organization) PSLV (Polar Satellite Launch Vehicle) launcher. The UVIT payload doors were opened on 30 November, 2015 and a 6 month performance verification (PV) phase began, ending in March 2016. The ground calibration of the payload has been discussed by Postma et al. (2011) with in-flight tests by Subramaniam et al. (2016).

There are now a complete set of PV phase observations which have been used to characterize the instrument (Tandon et al., 2016) with in-flight calibration and verification done by Tandon et al. (2017) and Rahna et al. (2017), and data are being released to the observers. However, there are still (at the date of writing) issues with the UVIT pipeline and there are data sets which cannot be processed. More importantly from our viewpoint is that the source code is proprietary and difficult to modify.

\footnotetext{
* Corresponding author

Email address: margarita.safonova62@iiap.res.in (Margarita Safonova)
}

We have chosen to create an alternative set of routines (JUDE: Jayant's UVIT Data Explorer) released under an Apache License ${ }^{1}$ which may be freely used and modified. JUDE begins with the Level 1 data provided by the Indian Space Science Data Centre (ISSDC) and produces photon lists and images suitable for scientific analysis.

It should be stressed that this package is not intended to be a replacement for the official UVIT pipeline but is rather a tool to examine the data in more detail. However, the authors are using JUDE for their own scientific purposes, and are actively maintaining and modifying the software as the instrument characterization improves. JUDE is archived at the Astrophysics Source Code Library (Murthy et al. 2016) and the latest version is available on GitHub (https://github.com/jaymurthy/JUDE). We solicit feedback on its operation and further improvements.

\section{Instrumentation and Observations}

The UVIT instrumentation has been described in Tandon et al. (2016), with a description of the entire AstroSat mission by Singh et al. (2014). The

\footnotetext{
${ }^{1}$ http://www.apache.org/licenses/LICENSE-2.0
} 
UVIT instrument consists of two 35-cm RitcheyChrétien telescopes with three intensified CMOS detectors. One telescope feeds a far ultraviolet (FUV) detector $(1300-1800 \AA)$, with the other feeding two detectors in the near ultraviolet (NUV: $1800-3000 \AA$ ) and visible (VIS: $3200-5500 \AA$ ), respectively, through a dichroic filter. A filter wheel in front of each detector allows different spectral ranges to be selected. The visible channel is not intended for science purposes and is used solely to track the spacecraft motion. Further information about the AstroSat mission and the instruments aboard may be obtained from the AstroSat Science Support Cell (http://astrosatssc.iucaa.in).

The UVIT instrument has observed a number of targets during the PV phase, where an observation may be broken into several exposures of several hundred seconds in length. UVIT has now begun regular observations and is shifting to an observer driven mission with proposals from the Indian and international astronomical community. The official UVIT pipeline is tasked with producing scientifically usable images, including flat-fielding, distortion correction, drift correction, and absolute calibration from the raw data, and is available from the Science Support Cell referenced above.

\section{JUDE}

\subsection{Overview}

We have written the JUDE software system entirely in the GNU Data Language (Coulais et al. 2010). GDL was chosen for the development environment because it is an interpreted language which lends itself to the interactive analysis of data. This was invaluable in the development of the pipeline where we could check each step and run commands interactively. GDL is an open source version of the Interactive Data Languag $\AA^{2}$ (IDL) and will run on a wide variety of systems, as will IDL. It will run most IDL programs without modification (and vice versa) allowing access to the rich library of utilities developed for IDL over the last four decades, easing the development of JUDE. We have tested JUDE using both GDL (version 0.9.6) and IDL with identical results on multiple operating systems. In the remainder of the paper, GDL and IDL may be freely interchanged.

The two UV channels typically operate in photon counting mode with the VIS camera operating in integrating mode. The primary science from UVIT will come from the two UV imagers with the

\footnotetext{
${ }^{2}$ http://www.harrisgeospatial.com
}

VIS camera intended solely to correct for spacecraft motion. We have therefore focussed on producing scientific products from the two UV channels.

Our starting point is the Level 1 data provided by the ISSDC from which we produce photon lists and images of the sky which may be used for further scientific analysis (Level 2 data). We have tested the JUDE system by reducing the entire set of PV observations and are now using it to analyse our GT (Guaranteed Time) observations. In the following sections, we will first describe the Level 1 and 2 data files, the operation of the pipeline and the logic behind the primary components of $J U D E$. The individual modules in JUDE are listed in Table 1. Each module is self-documenting with a prologue containing a description of the inputs and outputs. We use a number of routines from the IDL Astronomy Library (Landsman, 1995) without modification Appendix A: Table A.4 and the MPFIT routines (Markwardt, 2009) for PSF fitting.

\subsection{Level 1 Input Files}

Data from the AstroSat spacecraft are transmitted to the AstroSat Data Centre at the ISSDC where they are separated by instrument and written into Level 1 data files. They are then sent to the Payload Operations Centre (POC) at the Indian Institute of Astrophysics (IIA) where they will be processed by the official UVIT pipeline into Level 2 data products, suitable for scientific analysis. After validation, the Level 2 products will be sent back to the ISSDC which has the primary responsibility for data archival and dissemination.

The Level 1 data are distributed as a single zipped archive (earlier tar.gz) for each observation. The files within the archive are in a number of subdirectories organized by orbit number and type of file, all under a single top level directory. All data files are FITS binary tables (Pence et al. 2010) with individual frames tagged by mission time and containing, depending on the file, either housekeeping or science data. Due to the manner in which the data are read and transmitted to the ground, an archive may consist of multiple housekeeping and science files, possibly with duplicated frames and/or broken across multiple files.

We extracted all the files from the archive using unzip (or tar -xf) into a single top-level directory, which we use four sets of files (irrespective of subdirectory):

1. Level 1 data files for VIS ("uvtV"): Used to correct for spacecraft motion. Not required for pipeline operation. 
Table 1: JUDE modules

\begin{tabular}{|c|c|}
\hline Module & Purpose \\
\hline \multicolumn{2}{|r|}{ Accessory Programs } \\
\hline $\begin{array}{l}\text { jude_get_files } \\
\text { jude_params } \\
\text { jude_err_process }\end{array}$ & $\begin{array}{l}\text { Returns names of data files. } \\
\text { Sets operating parameters. } \\
\text { Error handler. }\end{array}$ \\
\hline \multicolumn{2}{|r|}{ Level 1 Data } \\
\hline $\begin{array}{l}\text { jude_read_vis } \\
\text { jude_create_uvit_hdr } \\
\text { jude_read_hk_files } \\
\text { jude_set_dqi } \\
\text { jude_get_xy } \\
\text { jude_cnvt_att_xy } \\
\text { jude_check_bod }\end{array}$ & $\begin{array}{l}\text { Reads the visible data. } \\
\text { Creates FITS data header for Level } 2 \text { data files. } \\
\text { Reads housekeeping files. } \\
\text { Checks instrumental parameters. } \\
\text { Extracts individual events from Level } 1 \text { data. } \\
\text { Calculates } X \text { and } Y \text { shifts from boresight. } \\
\text { Check and reject bright object detection (BOD). }\end{array}$ \\
\hline \multicolumn{2}{|r|}{ Level 2 Data } \\
\hline $\begin{array}{l}\text { jude_register_data } \\
\text { jude_add_frames } \\
\text { jude_vis_shifts } \\
\text { jude_add_vis } \\
\text { jude_obs_log } \\
\text { jude_merge_files } \\
\text { jude_match_vis_offsets } \\
\text { jude_apply_cal } \\
\text { jude_centroid }\end{array}$ & $\begin{array}{l}\text { Corrects for spacecraft motion. } \\
\text { Combines individual frames into image. } \\
\text { Calculates spacecraft motion from visible data. } \\
\text { Adds visible frames together. } \\
\text { Creates observation log from Level } 2 \text { files. } \\
\text { Combines files with overlapping data. } \\
\text { Matches the visible offsets to the UV channels. } \\
\text { Applies photometric calibration to data } \\
\text { Tracks spacecraft motion through centroids of stars. }\end{array}$ \\
\hline \multicolumn{2}{|r|}{ Driver Routines } \\
\hline $\begin{array}{l}\text { jude_driver_vis } \\
\text { jude_driver_uv } \\
\text { jude_uv_cleanup } \\
\text { jude_interactive }\end{array}$ & $\begin{array}{l}\text { Chains individual programs to produce Level } 2 \text { files in the VIS. } \\
\text { Chains individual programs to produce Level } 2 \text { files in the UV. } \\
\text { Post processing of Level } 2 \text { data. } \\
\text { Interactive exploration of Level } 2 \text { data. }\end{array}$ \\
\hline
\end{tabular}

2. Level 1 data files for either FUV ("uvtF") or NUV ("uvtN"): At lease one file must be present.

3. Housekeeping files ("*.lbt"): Frames with no housekeeping information are rejected.

4. Attitude files of spacecraft boresight("**att"): Required for a preliminary estimate of pointing.

\subsection{Running JUDE}

\subsubsection{Pipeline Operation}

We have written $J U D E$ as a set of procedures which must be run from within the GDL (or IDL) language. Each procedure is designed to be run independently but will operate as a pipeline if chained together. We have written a shell program Appendix B which we have used to process the entire set of PV observations without active monitoring. These files may be further processed by the user to co-add different exposures of a single target, extract point sources, and for other scientific purposes.

The individual JUDE procedures are listed in Table 1 and their operation is controlled by a GDL data structure Appendix C) where the structure is defined in, and defaults are set by, jude_params.pro. The user may change the parameters on a global level by editing the parameter file or on the fly through the command line.

\subsection{VIS Processing}

Observations in the VIS channel are taken in integration mode where the $512 \times 512$-pixel CMOS chip is read once per second and the pixels stored in a binary FITS table where the data from each frame are in 261 rows of 1008 pixels each, first with increasing $x(0-511)$ and then increasing $y$ $(0-511)$. The final $944(261 \times 1008-512 \times 512)$ elements are left blank and should be skipped. We used jude_read_vis.pro to read each VIS frame (Fig. 1) and extracted the $x$ and $y$ positions of the stars in the field with the library routine find.pro (based on DAOPHOT: Stetson (1987)). The spacecraft motion is just the shifts between successive frames which we calculated and wrote into a text file using jude_vis_shifts.pro.

The NUV and VIS channels share a telescope with the NUV light reflected, and the VIS light transmitted, through a dichroic and the shifts for 


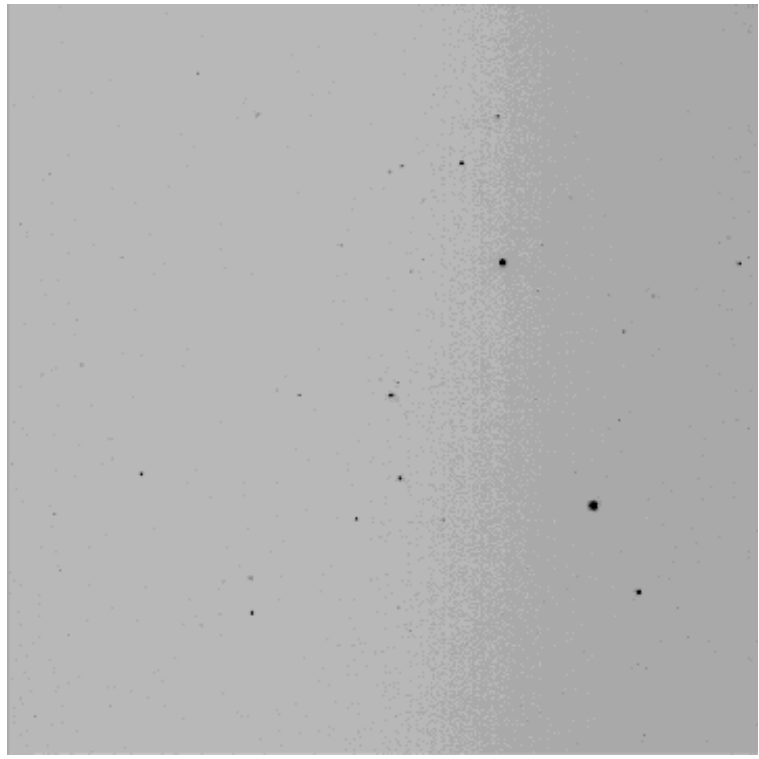

Figure 1: A 1 second frame of Holmberg II with the VIS imager.

the NUV image are mirrored around the $x$-axis

$$
\begin{aligned}
& x_{\mathrm{NUV}}=x_{\mathrm{VIS}}, \\
& y_{\mathrm{NUV}}=-y_{\mathrm{VIS}} .
\end{aligned}
$$

The FUV channel has its own telescope, and we found (empirically) that it is rotated from the VIS by $35^{\circ}$,

$$
\begin{aligned}
& x_{\mathrm{FUV}}=x_{\mathrm{VIS}} \cos \left(35^{\circ}\right)-y_{\mathrm{VIS}} \sin \left(35^{\circ}\right), \\
& y_{\mathrm{FUV}}=x_{\mathrm{VIS}} \sin \left(35^{\circ}\right)+y_{\mathrm{VIS}} \cos \left(35^{\circ}\right) .
\end{aligned}
$$

We use jude_match_vis_offsets.pro to store the offsets into the UV channels, using the spacecraft time as a reference. We have shown the original image with the spacecraft motion and the corrected image in Fig. 2. In practice, we find, as we will show below, that we can obtain better resolution through self-registration, i.e. using stars in the NUV (FUV) channel to correct for the motion in the NUV (FUV) channel.

\subsection{Housekeeping and Attitude}

The housekeeping files (*.lbt) contain a wealth of information about the health and the environmental conditions of the UVIT instrument, most of which are not relevant to our scientific analysis. The attitude files (*.att) contain the spacecraft boresight position as determined by the star sensor approximately every 16 seconds. We use jude_read_hk.pro to read the housekeeping and attitude files and jude_set_dqi.pro to match the housekeeping and attitude information with the sensor data based on the mission time. We perform basic sanity checks as part of our Level 1
Table 2: DQI values

\begin{tabular}{lll}
\hline DQI & Description & Module \\
\hline 0 & Data valid. & \\
1 & BOD & jude_check_bod \\
2 & Unused. & \\
4 & Unused. & jude_set_dqi \\
8 & No HK data. & jude_get_xy \\
16 & Duplicate frames. & jude_set_dqi \\
32 & Filter closed/invalid. & jude_set_dqi \\
64 & Voltage out of bounds. & jude_get_xy \\
128 & $>$ 1000 counts. & jude_get_xy \\
1024 & Parity violation. &
\end{tabular}

processing and set the DQI (data quality index) as in Table 2 Note that the DQI values are powers of two and additive; it is trivial to diagnose which data were rejected and why.

A bright object detection (BOD) sequence was run at the beginning of every observing sequence in which the gain was ramped up to the operating value. A few frames were sampled at each gain to ensure that there was no potential for damage to the instrument. Not all data files contain the BOD due to the manner in which the data were transmitted to the ground but we checked every observation and flagged those that were part of the BOD sequence.

\subsection{Processing UV Data}

The UVIT detectors are intensified $512 \times 512$ CMOS detectors (Tandon et al., 2016) read 29 times a second with an exposure time of 0.035 seconds per frame. Individual photons fall on the photocathode where they eject electrons that are accelerated through a microchannel plate (MCP) onto a phosphor screen. The resulting flashes of green light, each of which represents a single photon hit, are recorded on the CMOS sensor. Onboard software converts these flashes into individual photon events with a nominal resolution of $\frac{1}{8}$ of a pixel and packs each event into a byte array which is stored on-board and ultimately transmitted to the ground. The ISSDC separates the data by instrument and channel and passes them on to the user as Level 1 data files.

There is another mode in which a small $(100 \times$ 100 pixel) window is read at 600 frames per second as well as a transmission grating which produces low resolution spectra. The processing scheme is the same for those modes and we do produce usable data but have not tested the data products for scientific integrity.

Each event in the Level 1 data is stored as 6 bytes with two bytes for each of the $x$ and $y$ 


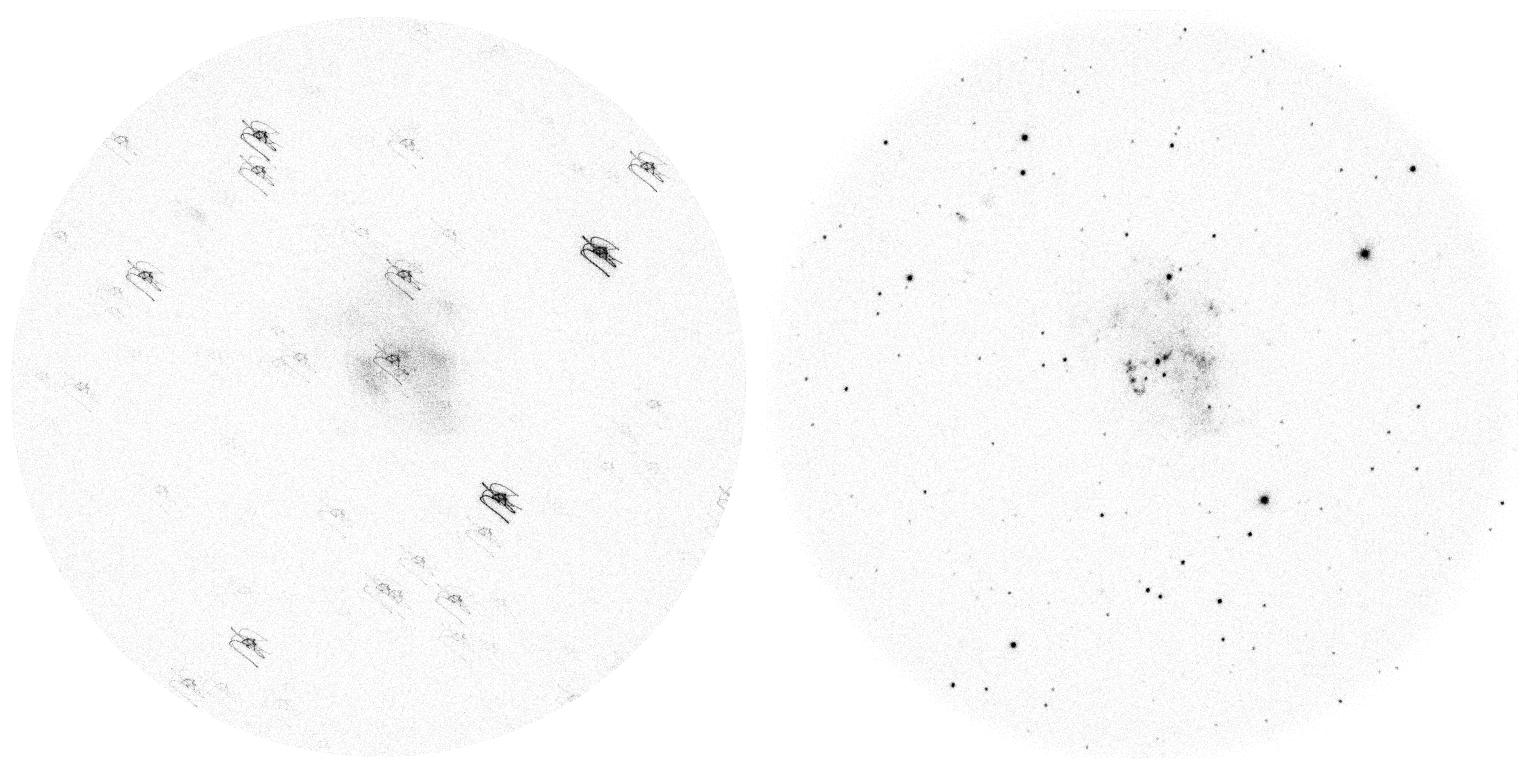

Figure 2: Holmberg II without correction for spacecraft motion on the left and after correction with the VIS offsets on the right.

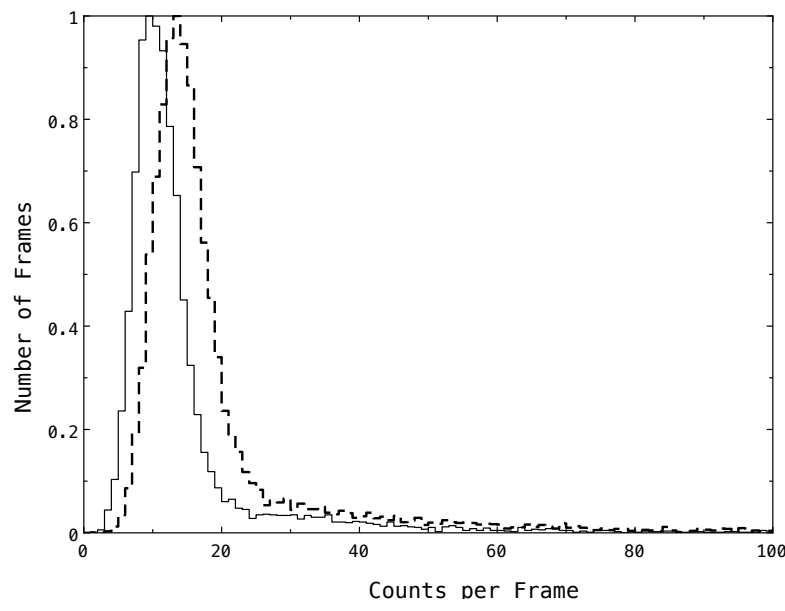

Figure 3: Normalised histogram of the count rate per frame in the FUV (solid line) and NUV (dashed line).

positions and the third for diagnostic information about that event (not used in JUDE). One frame contains a maximum of 336 events with further events resulting in a new frame. We read each frame and extracted the photon events using jude_get_xy.pro. The CMOS sensors are subject to cosmic ray hits giving rise to Cherenkov radiation within the MCP. This blue light results in a huge splash on the detector which is interpreted as a large number of individual photon hits by the onboard centroiding algorithm, all spurious. The actual count rate in one of our observations is plotted in Fig. 3 where the cosmic ray hits are represented by the long tail at higher count rates. The effect of including the spurious counts is to increase the overall background level in the image and we rec- ommend that they be discarded. The default in $J U D E$ is to define the threshold as $m+3 \sigma$ where $m$ is the median of the counts per frame over the entire observation and $\sigma=\sqrt{m}$ for the photon counting UVIT detectors. If desired, the threshold may be changed using the max_counts parameter (Table C.5). Note that we record no more than 1000 events per frame, a most unlikely level for any observation to reach.

Our final step in the production of Level 2 data was to remove duplicate frames and merge files which were broken in the middle of an exposure. This creates a number of FITS binary tables (Table 3 which contain the time-tagged photon lists with selected housekeeping data. These can be used for further scientific processing without returning to the Level 1 data and the housekeeping files, saving considerable time. One of the most time-consuming aspects of $J U D E$ is matching the event lists with the housekeeping data.

\subsection{Level 2 Output Files}

Our primary Level 2 data product is a UV photon list for every exposure (a contiguous set of UV data). This list is time-tagged and stored in a FITS binary table (Table 3). Each row of the FITS binary table represents a single 0.035 second frame identified by the time in seconds from Jan. 1,2010 . The $x$ and $y$ position of every photon in a frame are stored in two arrays ("X" and "Y") and the spacecraft motion is stored in "XOFF" and "YOFF". The output files from JUDE are described in Appendix D including the VIS output and the UV photon lists and images. 


\begin{tabular}{llll}
\hline & Variable & Data Type & Sample Value \\
\hline 1 & FRAMENO & LONG & 17007 \\
2 & ORIG_INDEX & LONG & 17451 \\
3 & NEVENTS & INT & 153 \\
4 & X & FLOAT & Array[1000] \\
5 & Y & FLOAT & Array[1000] \\
6 & MC & INT & Array[1000] \\
7 & DM & INT & Array[1000] \\
8 & TIME & DOUBLE & $1.8824165 \mathrm{e}+08$ \\
9 & DQI & INT & 0 \\
10 & FILTER & FLOAT & 271.232 \\
11 & ROLL_RA & DOUBLE & 11.761461 \\
12 & ROLL_DEC & DOUBLE & 85.226608 \\
13 & ROLL_ROT & DOUBLE & 166.56405 \\
14 & ANG_STEP & DOUBLE & 0.0030735851 \\
15 & XOFF & FLOAT & -0.998805 \\
16 & YOFF & FLOAT & -1.00348 \\
\hline
\end{tabular}

\subsection{Image Production}

In principle, it is easy to add the individual photon events from the Level 2 UV files to form an image of the sky, as we have done in Fig. 2 (using jude_add_frames.pro). However, the quality of the images will be determined largely by how well we are able to correct for the spacecraft motion. The VIS images were intended to track stars in the field and, as discussed in Section 3.4 we have matched the calculated VIS offsets with the UV frames to populate "XOFF" and "YOFF" in Table 3

If the VIS data are not available, we obtained a first estimate of the motion from the on-board star sensor which reports the spacecraft boresight (RA, Dec, Roll Angle) every 16 seconds (464 frames). We used these to shift and add frames and, in the same manner as the VIS data, found the shifts between successive frames. Most of the UV sources observed to date are relatively faint, largely to protect the detectors from damage, and we had to integrate over $100-200$ frames $(3.5-7$ seconds $)$ to get enough counts for find.pro to work and interpolate to find the shifts for each frame.

We found that we could dramatically improve the resolution if we adopted a more robust centroiding algorithm (jude_centroid.pro) in which we followed a single star in the image throughout the observation. The registration worked best when we added together $10-20$ frames with a star that had $3-10$ counts in that time. We calculated the centroid of the star in $x$ and $y$ in each set of frames and used the shifts between frames to derive the spacecraft motion. The difference between the spacecraft motion derived here and that inferred from the VIS channel is plotted in Fig. 4 and is on the order of 0.5 pixels (other than a constant

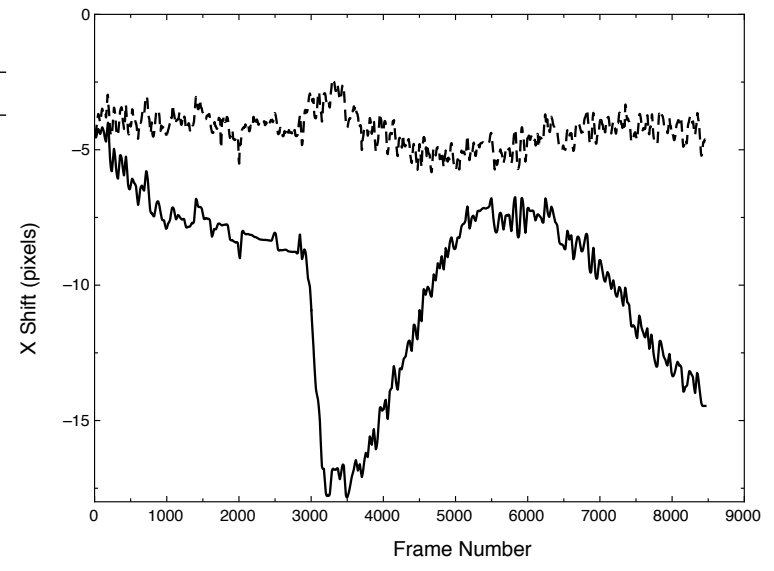

Figure 4: Spacecraft motion derived from the VIS channel (solid line). The jerk at about frame 3000 is likely due to the operation of the Scanning Sky Monitor (SSM). The difference between the motion derived from the UV channel and the VIS channel is plotted as a dashed line. The offset of about 5 pixels is due to differing starting points.

offset of 5 pixels due to a different choice for the reference frame). The better resolution obtained with jude_centroid.pro is apparent in Fig. 5 with more detail seen in the central portion of Holmberg II. The effective FWHM of the stars is $1.7^{\prime \prime}$ when we use jude_centroid, and $4.7^{\prime \prime}$ when we use the VIS offsets.

\section{Calibration}

We have used JUDE to perform an in-flight calibration of UVIT (Rahna et al. 2017) and have incorporated those results back in the programs. We have left the Level 2 data in units of counts per second but have added a field to the header with the scale factor for the appropriate conversion into energy units (ergs $\left.\mathrm{cm}^{2} \mathrm{~s}^{-1} \AA^{-1}(\mathrm{cps})^{-1}\right)$. We found no evidence for flat field variations, especially in view of the long star tracks due to the spacecraft motion. We have derived a distortion correction for the data but found that applying it did not improve the resolution. We therefore recommend that the distortion correction be done as part of the astrometric correction after the final image production.

The astrometric information in the header is based on the relatively poor attitude information from the star sensor. We made the decision that Astrometry.net (Lang et al. 2010) has solved this problem and, in keeping with our philosophy of reusing existing components, recommend it to the user for precise astrometry. 

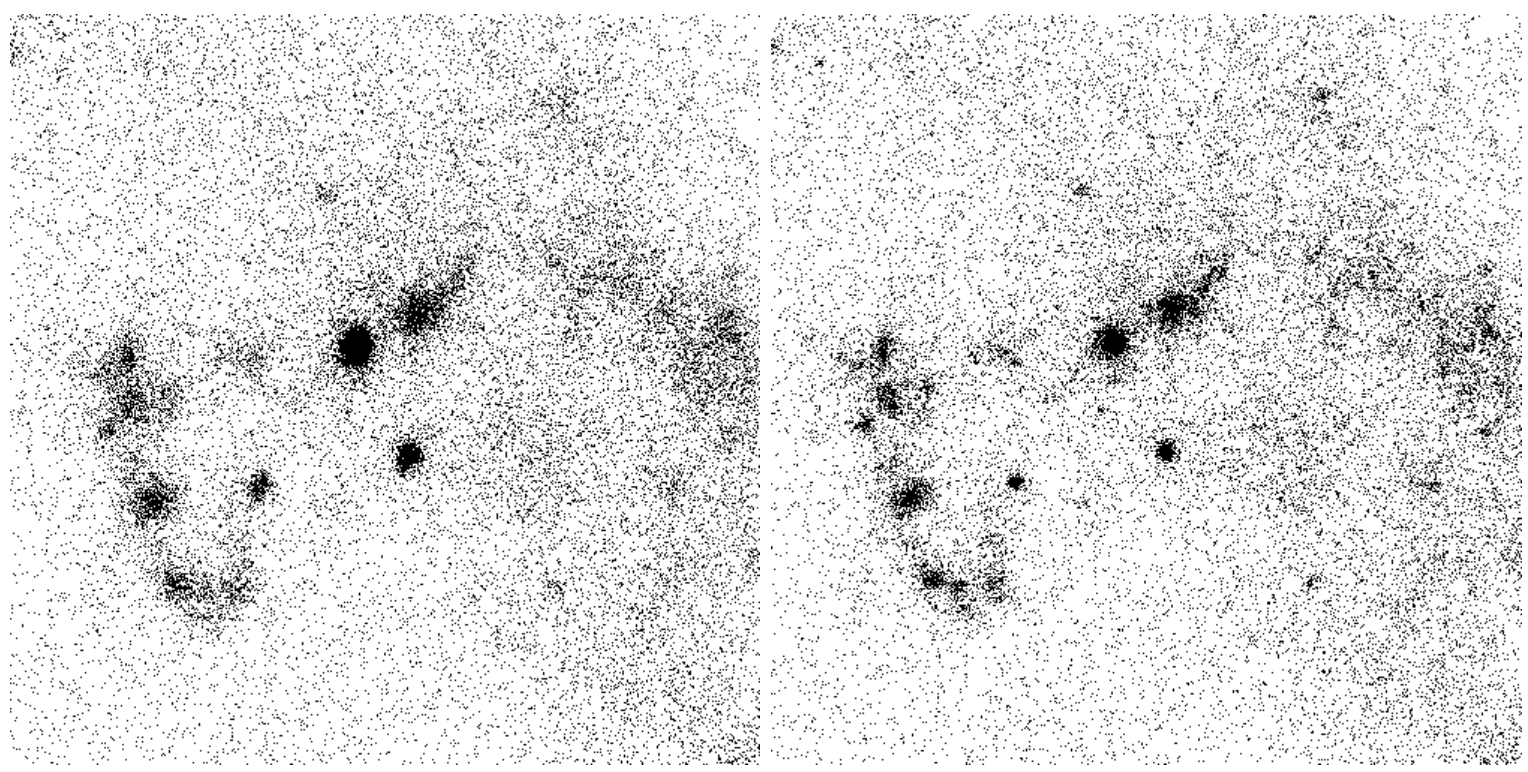

Figure 5: We achieve better resolution when we use self-registration through jude_centroid (right) than with the VIS-derived spacecraft motion (left).

\section{Conclusions}

We have designed a set of software routines that begin with Level 1 UVIT data from the ISSDC and produces images suitable for scientific purposes. We have tested the software on all our GT observations, and have produced Level 2 event lists and images for all observations through an automated process. A few observations required manual intervention, primarily due to registration problems. We have used JUDE to characterize the in-flight performance of UVIT (Rahna et al., 2017) and now expect to move on to the scientific exploitation of the data.

We have released JUDE under the Apache License 2.0 3 and it is available on the Astrophysics Source Code Library (Murthy et al. 2016) and on GitHub (https://github.com/jaymurthy/JUDE). We are using data from the UVIT for our science and, therefore, will continue to maintain and improve $J U D E$ as needed.

As a final note, the scientific exploitation of the UVIT data has been limited till now owing to the delays in the UVIT pipeline. We hope that with the release of JUDE to the astronomical community at large, more and better scientific results from the UVIT will be published. The only valid test of scientific software is if it is used widely, and we will continue to update the routines as we find errors or as they are reported to us. We welcome feature requests to improve the scientific utility of the programs.

\footnotetext{
${ }^{3}$ https://www.apache.org/licenses/LICENSE-2.0.txt
}

\section{Acknowledgements}

JM thanks John, Paul, George and Ringo for inspiration and the fortitude for dealing with the intricacies of dealing with a major mission. Many people at IIA, ISRO, IUCAA, TIFR, NRC (Canada) and Univ. of Calgary have contributed to different parts of the spacecraft, instrument and the operations. Drs. R. Mohan and J. Postma answered many questions about the data. We also acknowledge the Gnu Data Language (GDL), the IDL Astronomy Library and its many contributors. This research has made use of NASA Astrophysics Data System Bibliographic Services. We thank the referees for a thorough review that we believe has significantly improved the readability of the paper.

This research has been supported by the Department of Science and Technology under Grants No. SR/S2/HEP-050/2012 dated 14-08-2013 to Christ University and EMR/2016/00145 to IIA.

\section{References}

\section{References}

Coulais, A., Schellens, M., Gales, J., Arabas, S., Boquien, M., Chanial, P., Messmer, P., Fillmore, D., Poplawski, O., Maret, S., Marchal, G., Galmiche, N., Mermet, T., Dec. 2010. Status of GDL - GNU Data Language. In: Mizumoto, Y., Morita, K.-I., Ohishi, M. (Eds.), Astronomical Data Analysis Software and Systems XIX. Vol. 434 of Astronomical Society of the Pacific Conference Series. p. 187.

Landsman, W. B., 1995. The IDL Astronomy User's Library. In: Shaw, R. A., Payne, H. E., Hayes, J. J. E. 
(Eds.), Astronomical Data Analysis Software and Systems IV. Vol. 77 of Astronomical Society of the Pacific Conference Series. p. 437.

Lang, D., Hogg, D. W., Mierle, K., Blanton, M. Roweis, S., May 2010. Astrometry.net: Blind Astrometric Calibration of Arbitrary Astronomical Images. Astronom. J. 139, 1782-1800.

Markwardt, C. B., Sep. 2009. Non-linear Least-squares Fitting in IDL with MPFIT. In: Bohlender, D. A., Durand, D., Dowler, P. (Eds.), Astronomical Data Analysis Software and Systems XVIII. Vol. 411 of Astronomical Society of the Pacific Conference Series. p. 251.

Murthy, J., Rahna, P. T., Safonova, M., Sutaria, F., Gudennavar, S. B., Bubbly, S. G., Jul. 2016. JUDE: An Utraviolet Imaging Telescope pipeline. Astrophysics Source Code Library.

Pati, A. K., 1999. Proposed UV - optical payload for the Indian astronomy satellite. Bulletin of the Astronomical Society of India. 27, 295.

Pati, A. K., Rao, N. K., Aug. 1998. UV imaging telescope for the Indian Multiwavelength Astronomy Satellite. In: Bely, P. Y., Breckinridge, J. B. (Eds.), Space Telescopes and Instruments V. Vol. 3356 of Proc. SPIE. pp. 635640.

Pence, W. D., Chiappetti, L., Page, C. G., Shaw, R. A., Stobie, E., Dec. 2010. Definition of the Flexible Image Transport System (FITS), version 3.0. Astron. Astrophys. 524, A42.

Postma, J., Hutchings, J. B., Leahy, D., Jul. 2011. Calibration and Performance of the Photon-counting Detectors for the Ultraviolet Imaging Telescope (UVIT) of the Astrosat Observatory. Publ. Astr. Soc. Pac. 123, 833-843.

Rahna, P. T., Murthy, J., Safonova, M., Sutaria, F., Gudennavar, S. B., Bubbly, S. G., 2017. Investigating the In-Flight Performance of the UVIT Payload on AstroSat. E-print arXiv:1706.02523.

Singh, K. P., Tandon, S. N., Agrawal, P. C., Antia, H. M., Manchanda, R. K., Yadav, J. S., Seetha, S., Ramadevi, M. C., Rao, A. R., Bhattacharya, D., Paul, B., Sreekumar, P., Bhattacharyya, S., Stewart, G. C., Hutchings, J., Annapurni, S. A., Ghosh, S. K., Murthy, J., Pati, A., Rao, N. K., Stalin, C. S., Girish, V., Sankarasubramanian, K., Vadawale, S., Bhalerao, V. B., Dewangan, G. C., Dedhia, D. K., Hingar, M. K., Katoch, T. B., Kothare, A. T., Mirza, I., Mukerjee, K., Shah, H., Shah, P., Mohan, R., Sangal, A. K., Nagabhusana, S., Sriram, S., Malkar, J. P., Sreekumar, S., Abbey, A. F., Hansford, G. M., Beardmore, A. P., Sharma, M. R., Murthy, S., Kulkarni, R., Meena, G., Babu, V. C., Postma, J., Jul. 2014. ASTROSAT mission. In: Space Telescopes and Instrumentation 2014: Ultraviolet to Gamma Ray. Vol. 9144 of Proc. SPIE. p. 91441S.

Stetson, P. B., Mar. 1987. DAOPHOT - A computer program for crowded-field stellar photometry. Publ. Astr. Soc. Pac. 99, 191-222.

Subramaniam, A., Tandon, S. N., Hutchings, J., Ghosh, S. K., George, K., Girish, V., Kamath, P. U., Kathiravan, S., Kumar, A., Lancelot, J. P., Mahesh, P. K., Mohan, R., Murthy, J., Nagabhushana, S., Pati, A. K., Postma, J., Rao, N. K., Sankarasubramanian, K., Sreekumar, P., Sriram, S., Stalin, C. S., Sutaria, F., Sreedhar, Y. H., Barve, I. V., Mondal, C., Sahu, S., Jul. 2016. In-orbit performance of UVIT on ASTROSAT. In: Space Telescopes and Instrumentation 2016: Ultraviolet to Gamma Ray. Vol. 9905 of Proc. SPIE. p. 99051F.

Tandon, S. N., Hutchings, J. B., Ghosh, S. K., Subramaniam, A., Koshy, G., Girish V, V., Kamath, P. U., Kathiravan, s., Kumar, A., Lancelot, J. P., Mahesh, P. K., Mohan, R., Murthy, J., Nagabhushana, S., Pati A, A. K., Postma, J., Kameswara Rao, N., Sankarasubramanian, K., Sreekumar, P., Sriram, S., Stalin, C. S., Sutaria, F.
Sreedhar, Y. H., Barve, I. V., Mondal, C., Sahu, S., Dec. 2016. In-orbit Performance of UVIT and First Results. E-print arXiv:1612.00612.

Tandon, S. N., Subramaniam, A., Girish, V., Postma, J., Sankarasubramanian, K., Sriram, S., Stalin, C. S., Mondal, C., Joseph, S. S. P., Hutchings, J., Ghosh, S. K., Barve, I. V., George, K., Kamath, P. U., Kathiravan, S., Kumar, A., Lancelot, J. P., Mahesh, P. K., Mohan, R., Nagabhushana, S., Pati, A. K., Kameswara Rao, N., Sreedhar, Y. H., Sreekumar, P., Sutaria, F., May 2017. In-orbit Calibrations of the Ultra-Violet Imaging Telescope. E-print arXiv:1705.03715.

\section{Appendix A. IDL External Modules}

$J U D E$ uses routines from the IDL Astronomy Library (Landsman, 1995) and from the MPFIT library (Markwardt, 2009). These are listed in Table A.4 and may be downloaded individually or as part of the entire library.

\section{Appendix B. Running the pipeline}

We have written a GDL command file (process_uvit.com) to call each of the programs in order. The user will have to change the first two lines to conform to their environment.

1. 'path='jude_dir:'+!path - !path is a system variable specifying the directories where the programs are located, if not in the working directory. It should include JUDE files and any additional programs required.

2. dname='root_dir/' — Sets the variable dname to the root directory for the UVIT Level 1 data files.

3. jude_driver_vis,dname - Processes the VIS files and derives the spacecraft motion. The VIS processing should occur before the two UV channels because the image correction comes from the VIS channel.

4. jude_driver_uv,dname,/nuv - Processes the NUV files to produce event lists.

5. jude_driver_uv,dname,/fuv — Processes the FUV files to produce event lists. The two UV routines may be run in either order.

6. jude_uv_cleanup,/nuv — Merges the NUV event lists, matches with offsets from the VIS channel, and produces images.

7. jude_uv_cleanup,/fuv - Merges the FUV event lists, matches with offsets from the VIS channel, and produces images.

\section{Appendix C. Parameters}

The parameters used in $J U D E$ are listed in Table C.5 and are passed to the pipeline through a structure defined in jude_params.pro. The advantage of using a structure is that it allows for further parameters to be added if required, without changing the program invocation. 


\begin{tabular}{|c|c|}
\hline Module & Purpose \\
\hline add_distort.pro & Add the distortion parameters in an astrometry structure to a FITS header. \\
\hline ad2xy.pro & Coordinate to pixel conversion. \\
\hline cgErrorMsg.pro & a device-independent error messaging function. \\
\hline correl_images.pro & Compute the 2-D cross-correlation function of two images. \\
\hline corrmat_analyze.pro & Find the optimal $(x, y)$ offset to maximize correlation of 2 images. \\
\hline detabify.pro & Replace tabs with spaces. \\
\hline data_chk.pro & Checks input data. \\
\hline datatype.pro & Returns data type of a variable. \\
\hline daycnv.pro & Converts Julian dates to Gregorian calendar dates. \\
\hline gcirc.pro & Computes rigorous great circle arc distances. \\
\hline getcoords.pro & Converts a string with angular coordinates to floating point values. \\
\hline get_date.pro & Return the UTC date in CCYY-MM-DD format. \\
\hline get_equinox.pro & Returns equinox. \\
\hline getopt.pro & Convert a string into a valid scalar or vector. \\
\hline gettok.pro & String manipulation. \\
\hline get_wrd.pro & Return the nth word from a text string. \\
\hline file_exist.pro & Tests for the existence of a file. \\
\hline find.pro & Find point sources. \\
\hline file_stat.pro & Returns information on a file. \\
\hline fxaddpar.pro & Add parameter to FITS header. \\
\hline fxbhmake.pro & Create basic FITS header. \\
\hline fxhclean.pro & Removes keywords from FITS header. \\
\hline fxpar.pro & Obtains value of a parameter in the FITS header. \\
\hline fxparpos.pro & Finds position to insert par. \\
\hline fxposit.pro & FITS file manipulation. \\
\hline fxmove.pro & Skip extensions in a FITS file. \\
\hline make_astr.pro & Builds astrometry structure. \\
\hline match.pro & Find subscripts where vectors match. \\
\hline mean.pro & Find mean. \\
\hline mkhdr.pro & Makes FITS header. \\
\hline mpfit.pro & Perform Levenberg-Marquardt least-squares minimization. \\
\hline mpfit2dfun.pro & Perform Levenberg-Marquardt least-squares fit to a 2-D IDL function. \\
\hline mpfit2dpeak.pro & Fit a gaussian, lorentzian or Moffat model to data. \\
\hline mrd_hread.pro & Reads FITS header. \\
\hline mrdfits.pro & Reads FITS files. \\
\hline mrd_skip.pro & Skip bytes in a file. \\
\hline mrd_struct.pro & Create structure. \\
\hline mwrfits.pro & Writes FITS files. \\
\hline putast.pro & Adds astrometry to FITS header. \\
\hline quadterp.pro & Interpolation. \\
\hline remove.pro & Remove elements from vector. \\
\hline reverse.pro & Reverse the order of rows or columns in an array. \\
\hline srcor.pro & Match point sources in image. \\
\hline strsplit.pro & STRTOK wrapper. \\
\hline sxaddhist.pro & Adds HISTORY to FITS header. \\
\hline sxaddpar.pro & Adds parameter to FITS header. \\
\hline sxpar.pro & Obtain the value of a parameter in a FITS header \\
\hline tag_exist.pro & Check for tags in a structure. \\
\hline valid_num.pro & Checks that a string is a valid number representation. \\
\hline wcs_check_ctype.pro & Checks CTYPE parameters and return the projection type and coordinate type. \\
\hline wcs_rotate.pro & Rotate between standard and native coordinates. \\
\hline wcssph2xy.pro & Coordinate to pixel conversion. \\
\hline write_png.pro & Writes PNG (Portable Network Graphics) file. \\
\hline zparcheck.pro & Check type and size of a parameter. \\
\hline
\end{tabular}


Table C.5: Program parameters

\begin{tabular}{|c|c|c|}
\hline Parameter & Description & Default Value \\
\hline resolution & Number of sub-pixels in one physical pixel $(1,2,4$ or 8$)$. & 8 \\
\hline min_counts & Frames with less counts are rejected (Fig. 3). & 0 \\
\hline max_counts & $\begin{array}{l}\text { Frames with more counts are rejected. If } 0 \text {, we use the } \\
\text { median }+3 \times \sqrt{\text { median. }}\end{array}$ & 0 \\
\hline min_frame & $\begin{array}{l}\text { Starting frame. In practice, the first frame where the DQI } \\
\text { value is } 0 .\end{array}$ & 0 \\
\hline max_frame & Ending frame. If 0 , last frame where DQI $=0$ & 0 \\
\hline coarse_bin & Number of frames binned to find stars in jude_register.pro. & 200 \\
\hline fine_bin & $\begin{array}{l}\text { Number of frames binned to find centroids in } \\
\text { jude_centroid.pro. }\end{array}$ & 20 \\
\hline ps_threshold_fuv & $\begin{array}{l}\text { Threshold for FUV point sources in counts } \mathrm{s}^{-1} \text { pixel }^{-1} \text {. } \\
\text { Used in jude_register. }\end{array}$ & $3 \times 10^{-4}$ \\
\hline ps_threshold_nuv & $\begin{array}{l}\text { Threshold for NUV point sources in counts } \mathrm{s}^{-1} \text { pixel }^{-1} \text {. } \\
\text { Used in jude_register. }\end{array}$ & $1.5 \times 10^{-3}$ \\
\hline flat_field & $\begin{array}{l}\text { Place holder if we want to use a flat field. Not implemented } \\
\text { as yet. }\end{array}$ & "No flat field" \\
\hline events_dir & $\begin{array}{l}\text { Output directory for Level } 2 \text { event lists. Separated by } \\
\text { channel (VIS, NUV, FUV). }\end{array}$ & "events/" \\
\hline image_dir & Output directory for FITS images. & "images/" \\
\hline mask_dir & $\begin{array}{l}\text { Holds mask files. Each mask is a FITS file containing an } \\
512 \times 512 \text { array where the region of interest is marked with } \\
1 \text { and the other elements are } 0 \text {. Masks are not used in the } \\
\text { pipeline but the functionality is in jude_interactive.pro and } \\
\text { the masks are written by jude_mask.pro. }\end{array}$ & "masks/" \\
\hline def_nuv_dir & Default directory for NUV channel. & "nuv/" \\
\hline def_fuv_dir & Default directory for FUV channel. & "fuv/" \\
\hline def_vis_dir & Default directory for VIS channel. & "vis/" \\
\hline png_dir & Output directory for PNG diagnostic images. & "png/ \\
\hline vis_L2_dir & Output directory for VIS files. & "vis_files/" \\
\hline vis_off_dir & Output directory for files with spacecraft motion. & "vis_off/" \\
\hline vis_add_dir & Output directory for VIS images. & "vis_add/" \\
\hline temp_dir & Directory for temporary files. & "jude_temp/" \\
\hline
\end{tabular}




\section{Appendix D. Level 2 Data Format}

Appendix D.1. VIS Files

1. A GDL save set containing three variables:

(a) GRID - Floating point array of dimensions $512 \times 512 \times N F R A M E S$. The VIS data consist of individual CMOS frames with $512 \times 512$ pixels with a total number of frames in the Level 1 data denoted by NFRAMES.

(b) TIMES - Double precision array with NFRAMES elements containing the UVIT time (the number of seconds since Jan. 1, 2010).

(c) DATA_HDR — String array with the header information from the Level 1 VIS file. The format is the standard GDL format for FITS headers with one line for each keyword in the original FITS header.

2. A text file containing the offsets between each frame in the VIS data and the first frame. The first line has two elements: the starting and ending time for that file and each successive line has 4 elements, with the first column being the reference time and the second being the time for that particular frame. The last two columns are the $X$ and $Y$ shifts of that frame with respect to the reference frame. The times are specified to 6 decimal places with the first 4 being significant, and the $X$ and $Y$ offsets are specified to 2 decimal places.

3. A FITS file with the co-added VIS data, including the correction for spacecraft motion.

\section{Appendix D.2. UV Data}

1. A FITS binary table for each of the two UV bands containing the photon event list as a function of frame number with relevant housekeeping and attitude information (Table 3). An optional second extension contains the offsets from the VIS data. The components of the binary table are as follows:

(a) FRAMENO is the frame number from the Level 1 data. A single frame may run across multiple lines if there are more than 336 events recorded, usually due to a cosmic ray hit. There may also be duplicated frames.

(b) ORIG_INDEX is the row number (beginning with 0 as in the GDL convention) in the original data file (recorded in the FITS header). ORIG_INDEX is $\geq$ than FRAMENO due to the repetition of frames in the Level 1 data. (c) NEVENTS is the total number of photon events in the frame with a maximum of value of 999 .

(d) $X$ is an array contains the column number of each event up to NEVENTS ranging from 0 to 511.875 .

(e) $Y$ is an array contains the column number of each event up to NEVENTS ranging from 0 to 511.875 .

(f) $\mathrm{MC}$ is the minimum corner value in the $5 \times 5$ on-board centroiding.

(g) $\mathrm{DM}$ is the (maximum - minimum) $5 \times 5$ corner value. MC and DM are reported as they may be used for diagnostics but are not used in JUDE.

(h) TIME is the elapsed mission time in seconds from Jan. 1, 2010.

(i) DQI is a diagnostic flag set by the pipeline. The values are defined in $\mathrm{Ta}$ ble 2

(j) FILTER is the angle of the filter wheel where the conversion from angle to filter is in jude_set_dqi.pro.

(k) ROLL_RA is the right ascension (RA) of the spacecraft boresight as reported by the star sensor.

(1) ROLL_DEC is the declination (DEC) of the spacecraft boresight as reported by the star sensor.

(m) ROLL_ROT is the roll angle of the spacecraft boresight as reported by the star sensor.

(n) ANG_STEP is the shift in the spacecraft pointing between one frame and the next in arcseconds.

(o) XOFF is the shift in sensor pixels (where the CMOS sensor is a $512 \times 512$ element array) between the frame and the reference frame in the $X$ direction, where the reference frame is defined by the FITS header of the image file.

(p) YOFF is the same as XOFF but in the $Y$ direction.

2. A FITS image file for each UV band with two extensions. The first extension is the coadded data from a single Level 1 file in units of counts s ${ }^{-1}$ pixel $^{-1}$ with the second extension containing the exposure time per pixel. Although the file contains astrometric information, it is only as accurate as the spacecraft star sensor. The scale factor to convert from counts s${ }^{-1}$ pixel $^{-1}$ into ergs $\mathrm{cm}^{-2} \mathrm{~s}^{-1} \AA^{-1}$ is in the FITS header.

3. A PNG file for a quick look containing the scaled image, a plot of the DQI values, a histogram of the number of counts, and a plot of the spacecraft motion. 
4. A text file with a comma separated observation $\log$. 